\title{
Erratum to: Measurement of badminton racket deflection during a stroke
}

\author{
Maxine Kwan • Ching-Lung Cheng • \\ Wen-Tzu Tang $\cdot$ John Rasmussen
}

Published online: 10 June 2010

(C) International Sports Engineering Association 2010

Erratum to: Sports Eng (2010) 12:143-153

DOI 10.1007/s12283-010-0040-5

Due to a processing error, the presentation of Eqs. 6-8, 14, and 16 was incorrect. The correct versions are given below:

$$
\begin{aligned}
& W_{n}(x)=\sin \beta_{n} x-\sinh \beta_{n} x-\alpha_{n}\left(\cos \beta_{n} x-\cosh \beta_{n} x\right) \\
& \cos \beta_{n} L \cdot \cosh \beta_{n} L=-1 \\
& \alpha_{n}=\frac{\sin \beta_{n} L+\sinh \beta_{n} L}{\cos \beta_{n} L+\cosh \beta_{n} L} \\
& W_{n}^{\prime \prime}(x)=-\beta_{n}^{2}\left(\sin \beta_{n} x+\sinh \beta_{n} x-\alpha_{n}\left(\cos \beta_{n} x+\cosh \beta_{n} x\right)\right) \\
& \varepsilon\left(x_{s g}, t\right)=-R W_{n}^{\prime \prime}\left(x_{s g}\right) \frac{2\left(\alpha_{n}+\beta_{n} h\right)}{b \omega_{n} \beta_{n}^{2}} \int_{0}^{t} \alpha_{\varepsilon}(\tau) \sin \omega_{n}(t-\tau) \mathrm{d} \tau \\
& \quad=-R W_{n}^{\prime \prime}\left(x_{s g}\right)\left(\alpha_{\varepsilon} * g\right)(t)
\end{aligned}
$$

The online version of the original article can be found under doi:10.1007/s12283-010-0040-5.

M. Kwan $(\bowtie) \cdot$ J. Rasmussen

Department of Mechanical Engineering, Aalborg University,

Pontoppidanstraede 101, 9220 Aalborg East, Denmark

e-mail:mak@me.aau.dk

URL: http://me.aau.dk

C.-L. Cheng · W.-T. Tang

Graduate Institute of Coaching Science,

National Taiwan Sport University, 250 Wen Hua 1st Rd,

Kweishan, Taoyuan 333, Taiwan, ROC 